\title{
WIDEBAND RF SYSTEM FOR THE FERMILAB RECYCLER RING*
}

\author{
J. E. Dey and D. W. Wildman, Fermilab, Batavia, IL
}

\begin{abstract}
A broadband RF system has been built for the new Fermilab Recycler Ring. It is designed to both bunch and capture beam during transfers and create multiple, moveable RF barrier buckets for azimuthal control of the stored antiproton beam. Beam will be bunched and captured using bursts of four RF cycles at $2.5 \mathrm{MHz}$. To maximize bucket area, the multiple barrier buckets will be generated by pairs of $2000 \mathrm{~V}$ bipolar square pulses (1 to 2 $\mu \mathrm{s}$ in duration). The high level portion of the system consists of four $50 \Omega$ ferrite loaded cavities, each driven by a $3.5 \mathrm{~kW}$ broadband $(10 \mathrm{kHz}-100 \mathrm{MHz})$ solid-state amplifier.
\end{abstract}

\section{INTRODUCTION}

The Fermilab Recycler Ring [1] is an $8 \mathrm{GeV} / \mathrm{c}$ antiproton storage ring, utilizing permanent magnets, located directly above the new Fermilab Main Injector. The Recycler will perform multiple functions in the Tevatron colliding beams program. Cooled antiprotons from the Accumulator will be periodically injected and stored in the Recycler. When the desired antiproton current is reached, four bunches of antiprotons will be formed in adjacent 2.5 $\mathrm{MHz}$ RF buckets and be extracted from the Recycler into the Main Injector. The Main Injector will then accelerate these bunches to $150 \mathrm{GeV} / \mathrm{c}$ and inject them into the Tevatron collider. This process will be repeated 9 times until a total of 36 bunches are circulating in the Tevatron.

After the luminosity of the Tevatron collider has decreased due to beam emittance growth, the proton and antiproton beams will be decelerated back down to 150 $\mathrm{GeV} / \mathrm{c}$, the protons will be scraped away, and the remaining antiproton bunches will be transferred, in groups of four, back into the Main Injector. Once in the Main Injector, the four bunches will be further decelerated to $8 \mathrm{GeV} / \mathrm{c}$ and injected into the Recycler to be cooled and reused in the collider. This process will be repeated a total of 9 times until all of the antiprotons that were initially in the Tevatron are transferred back into the Recycler.

\section{RF SYSTEM REQUIREMENTS}

At any given time, the Recycler ring may contain cooled beam, hot recycled beam from the Tevatron, beam being injected from the Accumulator, or beam being transferred either into or out of the Main Injector. During these processes, the various beam segments must be separated from each other azimuthally around the ring.

"Operated by the Universities Research Association, Inc. under contract with the U.S. Department of Energy.
In addition, newly injected beam has to be captured, debunched, and combined with the beam from earlier transfers. For transfers into the Main Injector, a fraction of the cooled beam has to be isolated and then bunched in $2.5 \mathrm{MHz}$ RF buckets. To accommodate all of these beam manipulations, a wideband RF system capable of generating three independent sets of barrier [2] RF buckets has been constructed. To maximize the barrier bucket area for a given azimuthal extent, the barrier buckets are formed by two bipolar rectangular pulses of adjustable width and amplitude. To generate the $2.5 \mathrm{MHz}$ buckets needed during transfers, the RF system will also produce a train of four RF cycles at $2.5 \mathrm{MHz}$ between two of the bipolar barrier buckets.

For a rectangular barrier bucket, formed by two pulses of width $\mathrm{T}$ and amplitude $+/-\mathrm{V}_{\mathrm{o}}$, the bucket half height is given by:

$$
\Delta \mathrm{E}_{1 / 2}=\sqrt{\frac{\mathrm{T}}{\mathrm{T}_{\mathrm{o}}} \frac{2 \beta^{2}}{\eta} \text { e } \mathrm{V}_{\mathrm{o}} \mathrm{E}_{\mathrm{o}}}
$$

where $E_{0}$ is the beam energy, $\eta$ is the momentum compaction factor, and $\mathrm{T}_{\mathrm{o}}$ is the revolution period. If the two rectangular barrier pulses of width $\mathrm{T}$ are allowed to touch, the resulting bucket area $\mathrm{A}$ is

$$
\mathrm{A}=\frac{8}{3} \Delta \mathrm{E}_{1 / 2} \mathrm{~T}
$$

To ensure that there is sufficient bucket area available to capture and confine the hot recycled beam from the Tevatron, the RF system must be capable of generating 1 to $2 \mu \mathrm{s}$ wide rectangular bipolar pulses with a peak voltage of $2 \mathrm{kV}$. Lee and $\mathrm{Ng}$ [3] have shown that the dynamics of a pair of these barrier pulses depend only on the total voltage integral of the barrier pulses and is independent of the actual RF waveform. This important fact relaxes many of the hardware requirements since the two halves of the barrier pulse pairs need not have equal amplitudes, be symmetric, or even have similar shapes.

\section{HARDWARE}

The high level RF system consists of four $50 \Omega$ cavities, each driven individually by an Amplifier Research model 3500A100 broadband solid-state amplifier. Each amplifier, operating in the push-pull mode, can supply a minimum of 3500 watts output power over the frequency range of $10 \mathrm{kHz}$ to $100 \mathrm{MHz}$. The amplifiers, located in the MI-60 service building, are connected to the cavities in the tunnel by $20 \mathrm{~m}$ of 7/8" Andrew LDF5-50 coaxial line. 


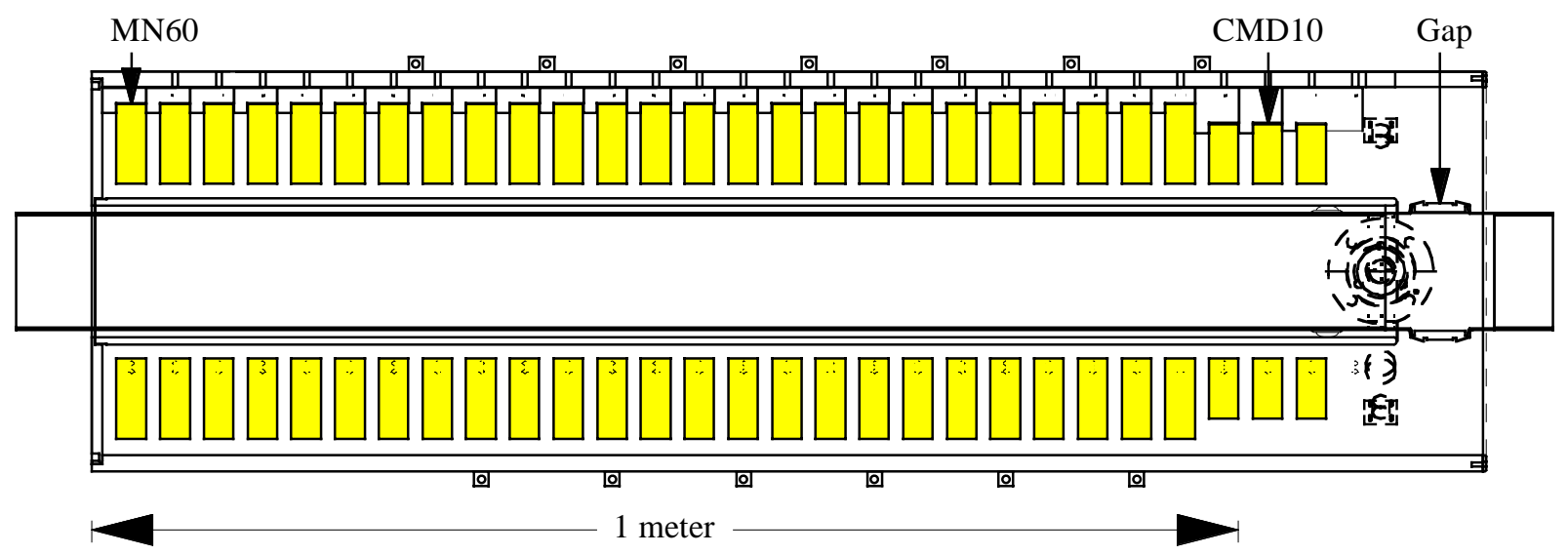

Figure 1: Schematic drawing of Recycler Wideband RF Cavity

A schematic drawing of the wideband cavity is shown in Figure 1. It consists of a water-cooled outer aluminum shell, an aluminum inner conductor, and a 4" diameter stainless steel beampipe with a 1" ceramic gap which is electrically connected to the cavity with beryllium-copper finger stock. A thin copper end plate completes the electrical conmnection between the beam pipe and outer cavity shell. The beampipe is thermally isolated from the cavity and is wrapped with heating tapes to allow the vacuum system to be baked out after installation. The cavity is filled with twenty-five, 11.5"OD x 6"ID x 1" thick Mn-Zn ferrite cores (Ceramic Magnetics MN60) and three, 10"OD x 6"ID x 1" thick Ni-Zn ferrite cores (Ceramic Magnetics CMD10). The ferrite cores are air-cooled, spaced by 0.5 ", and supported by Kapton spacer blocks. A 10 $\mathrm{kW}$, water-cooled, RF load; modified with a $60 \mathrm{ohm}$ resistor, is connected directly across the cavity gap. This resistor in parallel with the stack of ferrites results in an input impedance of approximately $50 \mathrm{ohms}$ over the frequency range of $100 \mathrm{kHz}$ to $20 \mathrm{MHz}$. Both the input drive and the $60 \Omega$ resistor are connected to the inner conductor at the gap by 1 " wide x 4 " long flat copper straps. The voltage developed across the gap is monitored by two 1000/1 resistive voltage dividers.

The low level RF drive signal is derived from eight, DSP controlled, arbitrary waveform generators synchronized to the machine revolution frequency of $89.8 \mathrm{kHz}$ [4]. A feedforward circuit is used to compensate for the droop induced by the ac coupled high power amplifier and the low frequency response of the RF cavity. The feedforward circuit utilizes an AD830 high speed video difference amplifier and a passive network that models the amplifier/cavity response to produce the modified drive signal. The modified low level signal is then amplified by a $2 \mathrm{kHz}-$ $500 \mathrm{MHz}$ amplifier (Mini-Circuits ZHL-6A), divided by resistive splitters, and then distributed with the proper delays to the four 3500 watt amplifiers.

Photographs of a wideband amplifier and cavity are shown in Figures 2 and 3. In Figure 2, four identical air cooled RF modules, which are combined to form its $3.5 \mathrm{~kW}$ output, are visible in the rear view. Figure 3 is a photograph of the cavity, taken in the laboratory, showing the outer shell aluminum cooling coils ,the $60 \Omega$ RF load, and the copper end plate connecting to the protruding beampipe.

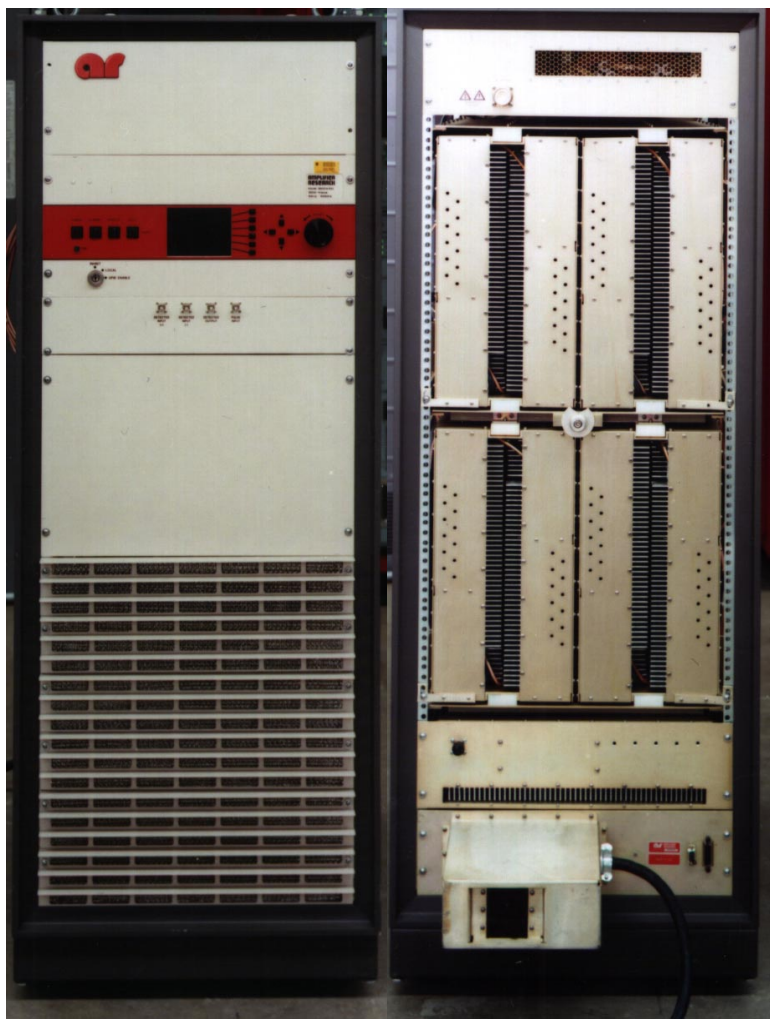

Figure 2: Front and Rear views of Amplifier Research model 3500A100. 


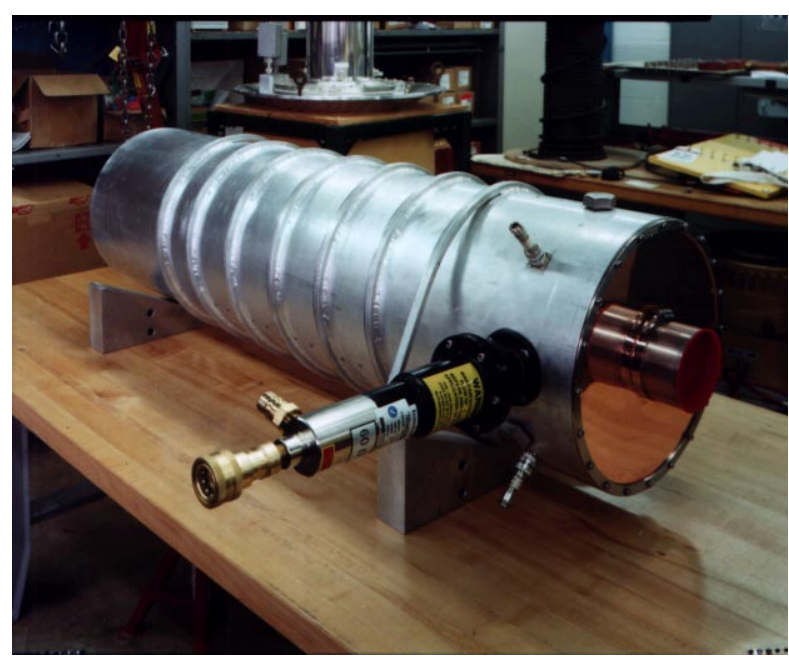

Figure 3: Wideband Cavity.

\section{RESULTS}

A network analyzer plot, from $50 \mathrm{kHz}$ to $20 \mathrm{MHz}$, of the real and imaginary parts of the wideband cavity input impedance is shown in Figure 4. This impedance represents the load seen by the high power RF amplifier. The upper trace shows the real impedance on a $2 \Omega /$ div scale centered around $52 \Omega$. The lower trace is the imaginary component centered at $0 \Omega$ with a 5 $\Omega /$ div scale. At low frequencies the cavity looks inductive. It is slightly capacitive between 0.5 and 4 $\mathrm{MHz}$ and then becomes inductive over the remaining frequency range.

Figure 5 shows the output signal from one of the four Recycler RF cavity gap monitors averaged over 16 machine revolutions. The data were taken without any beam in the machine with a final amplifier average power output of 2560 watts. Three sets of rectangular barrier pulses with different amplitudes and durations are shown along with four cycles of $2.5 \mathrm{MHz}$ for beam capture. The peak gap voltage is approximately +/500 volts. With all four RF systems operating, a combined total accelerating gap voltage of more than $+/-2 \mathrm{kV}$ will be available. Tests of the new RF system with beam are scheduled for late spring.
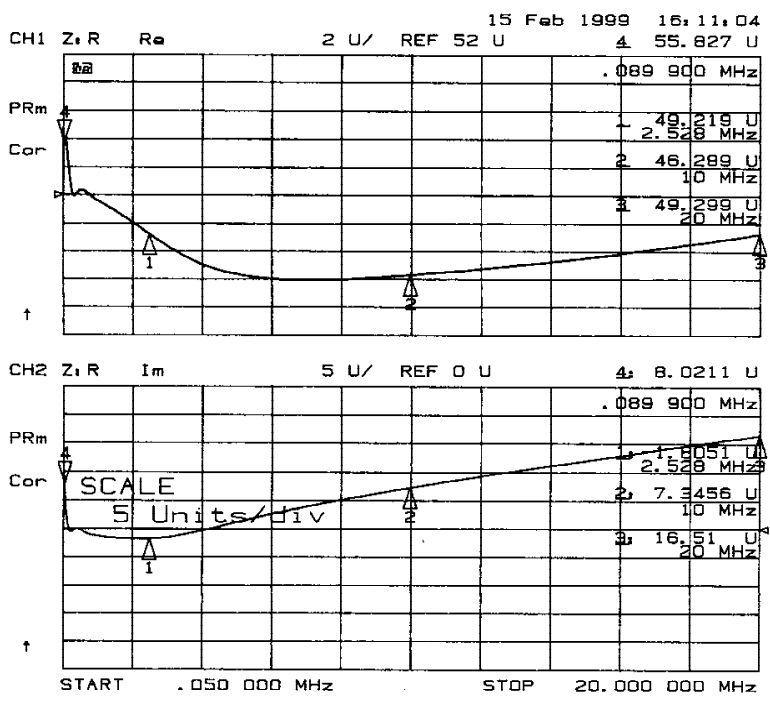

Figure 4: Input Impedance of Wideband Cavity.

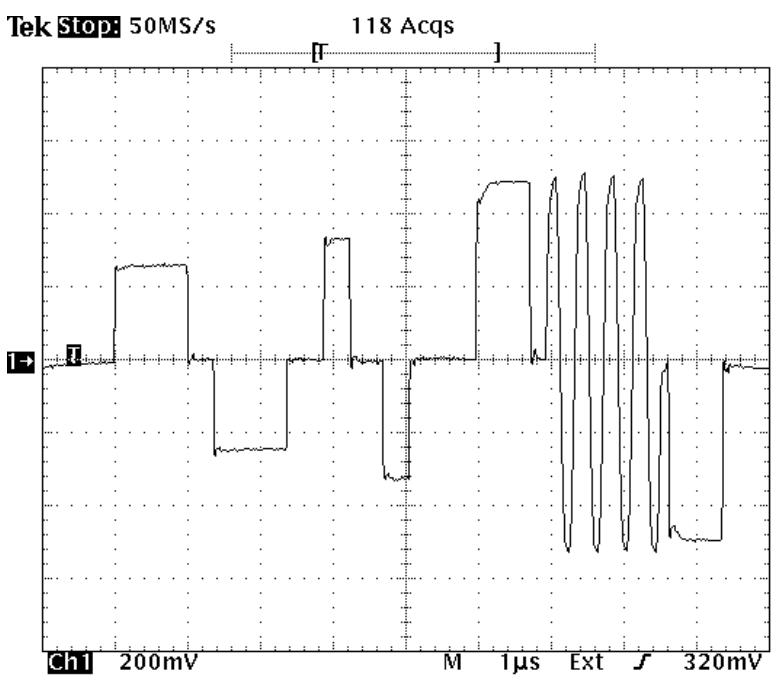

Figure 5: Gap voltage monitor output (1000V/V).

\section{REFERENCES}

[1] Fermilab Recycler Ring Technical Design Report, Fermilab (1996)

[2] J.E. Griffin, C. Ankenbrandt, J.A. Maclachlan, and A. Moretti, "Isolated Bucket RF Systems in the Fermilab Antiproton Facility", IEEE Trans. Nuc. Sci. NS-30, 3502 (1983)

[3] S.Y. Lee and K.Y. Ng, "Particle dynamics in storage rings with barrier rf systems", Phys. Rev. E55, 5992 (1997)

[4] B. Chase, A. Mason, and K. Meisner, "Current DSP Applications in Accelerator Instrumentation and RF", Proceedings of the 1997 Int. Conference on Accelerator and large Experimental Physics Control Systems, Beijing, China (1997) 